\title{
Production of cyathane type secondary metabolites by submerged cultures of Hericium erinaceus and evaluation of their antibacterial activity by direct bioautography
}

T. Shen ${ }^{1}$, G. Morlock ${ }^{2}$ and H. Zorn ${ }^{1 *}$

\begin{abstract}
Background: Fungi of the phylum Basidiomycota are well-known to form a broad spectrum of biologically active secondary metabolites, especially low molecular weight compounds such as terpenoids. Hericium erinaceus produces various cyathane type diterpenoids including erinacines. However, no quantitative data and production kinetics have been reported on the biosynthesis of the erinacines $C$ and $P$ in submerged cultures. In the present study, the production of erinacine $C$ was optimized, and the product formation kinetics as well as the antimicrobial activity were studied by high-performance liquid chromatography (HPLC), high-performance thin-layer chromatography (HPTLC) and direct bioautography.

Results: Oatmeal and Edamin ${ }^{\circledR}$ K were identified to be crucial media components for an efficient production of erinacine $C$. The highest concentrations of erinacine $C$ were obtained in the optimized culture medium on the $9^{\text {th }}$ culture day (approximately $260 \mathrm{mg} \mathrm{L}^{-1}$ ). The production of erinacine $P$ was strongly time dependent. The maximum concentration of erinacine P of $184 \mathrm{mg} \mathrm{L}^{-1}$ was observed on the third culture day. Afterwards, the concentrations of erinacine $P$ decreased while the concentrations of erinacine $C$ steadily increased. Comparable results were obtained by HPTLC with UV detection and HPLC with diode-array detection (DAD) analyses. Direct bioautography allowed for an additional analysis of the antimicrobial activity of the secondary metabolites.
\end{abstract}

Conclusions: The $\mathrm{C}$ and $\mathrm{N}$ sources oatmeal and Edamin ${ }^{\circledR} \mathrm{K}$ induced the formation of erinacine $\mathrm{C}$. Detailed product formation kinetics of the erinacines $C$ and $P$ have been reported for the first time. HPTLC combined with the Aliivibrio fischeri bioassay allowed for an instant detection of cyathane diterpenoids in crude extracts and for an evaluation of the antimicrobial activity of the secondary metabolites directly on the plate.

Keywords: Hericium erinaceus, Cyathane type diterpenoids, Erinacine C, Erinacine P, HPTLC, TLC-MS interface, Aliivibrio fischeri

\section{Background}

Basidiomycetes are the highest developed fungi. Many of them, if not all, synthesize biologically active compounds with medicinal properties in their fruiting bodies and mycelia. Hericium erinaceus is an edible mushroom that belongs to the family Hericiaceae and has been used for

\footnotetext{
*Correspondence: holger.zorn@uni-giessen.de

1 Institute of Food Chemistry and Food Biotechnology, Justus Liebig

University Giessen, Heinrich-Buff-Ring 17, 35392 Giessen, Germany

Full list of author information is available at the end of the article
}

the treatment of, e.g., digestive diseases in traditional Chinese medicine for more than 1000 years. Various compounds isolated from $H$. erinaceus, high molecular weight compounds as well as small molecules, have shown a variety of beneficial functions, such as anticancer, anti-inflammatory, and immunomodulatory properties [1-5]. Hericenones and erinacines are the major bioactive low molecular weight compounds formed by fruiting bodies and submerged cultures of $H$. erinaceus, respectively. Several of these compounds have been 
shown to significantly induce nerve growth factor (NGF) synthesis and to protect neuronal cells against endoplasmic reticulum (ER) stress- or oxidative stress-induced cell death. Therefore, the consumption of $H$. erinaceus, and of dietary supplements derived thereof, has been suggested for the prevention and treatment of dementia, and especially of Alzheimer's disease [1, 6-12].

The erinacines $\mathrm{A}$ to $\mathrm{I}, \mathrm{P}$, and $\mathrm{Q}$ are diterpenoid compounds with a cyathane skeleton consisting of five-, six-, and seven-membered rings. Among the erinacines A to G, erinacine C (Fig. 1) showed the strongest induction of NGF synthesis [1]. Various strategies have been reported for the chemical synthesis of cyathane type diterpenoids, e.g., the total synthesis of allocyathin $B_{2}$, erinacine $A$, and erinacine $E$ [13-17]. However, all of the chemical syntheses are complex multistep processes and suffer from low yields. Watanabe and Nakada [15] described the total synthesis of erinacine $\mathrm{E}$ in 39 steps with a total yield of $0.9 \%$. Snider [17] reported the total synthesis of erinacine A in 19 steps with a yield of $1 \%$. Therefore, the biotechnological production of erinacine $\mathrm{C}$ was investigated in submerged cultures of $\mathrm{H}$. erinaceus in the present study. The culture substrates were optimized and the production kinetics of erinacine $\mathrm{C}$ and of its supposed precursor erinacine $\mathrm{P}$ (Fig. 1) were recorded by HPLC-DAD analysis. Additionally, the combination of high-performance thin-layer chromatography (HPTLC) with a bioassay allowed for a fast evaluation of the antimicrobial activity of the erinacines.

\section{Results}

\section{Optimization of erinacine $C$ production}

The concentrations of erinacine $C$ formed in the mycelia and supernatants of the cultures grown in media 1-7 were different (Fig. 2). The presence of oatmeal was crucial for the biosynthesis of erinacine $C$, as erinacine $C$ was not detected in medium 3 (without oatmeal). Besides, the use of Edamin ${ }^{\circledR} \mathrm{K}$ increased the formation of erinacine $\mathrm{C}$ substantially. The concentration of erinacine $\mathrm{C}$ in medium 7 , in which Edamin ${ }^{\circledR} \mathrm{K}$ was used instead of Edamin ${ }^{\circledR} \mathrm{S}$, was about 8.5 times higher than in the reference medium 1.
Based on these results, the effect of oatmeal on erinacine $C$ production was rechecked in medium 7 (with oatmeal) versus medium 8 (without oatmeal). Erinacine $\mathrm{C}$ was produced in medium 8 in spite of the missing oatmeal, but the production of erinacine $C$ was higher in medium 7 (Fig. 3). Approximately $257 \mathrm{mg} \mathrm{L}^{-1}$ erinacine $\mathrm{C}$ was detected in the supernatants in medium 7 on the 9th culture day, compared to $\sim 85 \mathrm{mg} \mathrm{L}^{-1}$ in the supernatants of medium 8 . Therefore, medium 7 was chosen for the following experiments.

The concentrations of erinacine $\mathrm{P}$ and $\mathrm{C}$ were both found to be time-dependent (Fig. 4). The concentrations of erinacine $\mathrm{P}$ increased during the first three culture days and decreased thereafter. With the decreasing concentrations of erinacine $P$, the concentrations of erinacine $\mathrm{C}$ increased steadily. A maximum concentration of erinacine $\mathrm{P}$ of $\sim 184 \mathrm{mg} \mathrm{L}^{-1}$ was observed on the third culture day, while the maximum concentration of erinacine $\mathrm{C}$ was $257 \mathrm{mg} \mathrm{L}^{-1}$ on the 9th culture day.

\section{HPTLC-UV/vis/FLD-Aliivibrio fischeri analysis}

HPLC has been mainly applied for the analysis of natural products from complex matrices. However, the use of HPTLC for natural product analysis provides a number of advantages. It allows, e.g., for a screening for bioactivity directly on the plate, for quantification, and by coupling to mass spectrometers also for compound confirmation or structure suggestions.

The compound zones at $h R_{\mathrm{F}} 34$ (erinacine $\mathrm{P}$ ) and $h R_{\mathrm{F}}$ 49 clearly showed antibacterial activity (Fig. 5). Erinacine $\mathrm{P}$ was also detectable by inspection of the chromatogram at UV $254 \mathrm{~nm}$, while the substance at $h R_{\mathrm{F}} 49$ was fluorescent at UV $366 \mathrm{~nm}$. The results obtained by HPLC-DAD were clearly confirmed by HPTLC-UV/ FLD analysis followed by bioautography. Also here, the concentrations of erinacine P increased during the first 3 cultivation days and decreased thereafter. It was evident that the unknown bioactive compound zones at $h R_{\mathrm{F}} 49$ clearly increased, similarly to erinacine $C$ as discussed before.

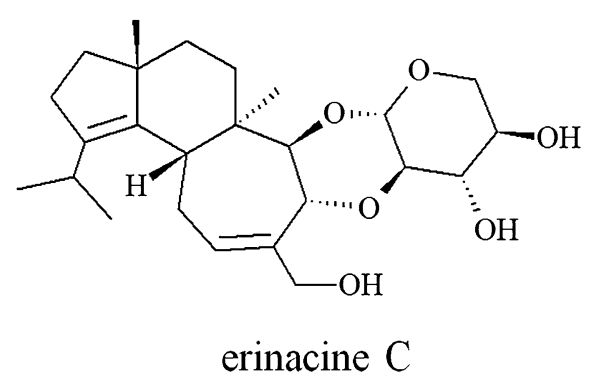

Fig. 1 Structures of erinacine $C$ and $P$

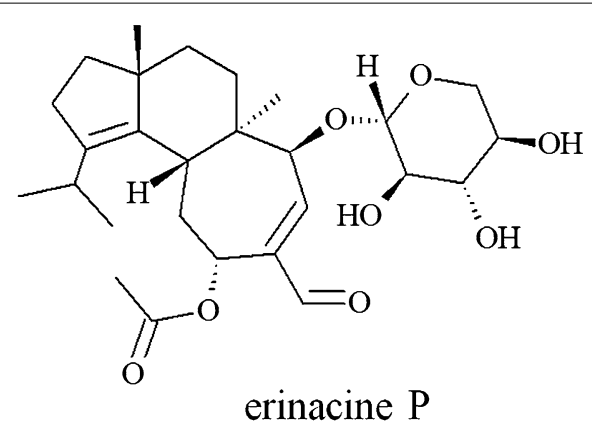



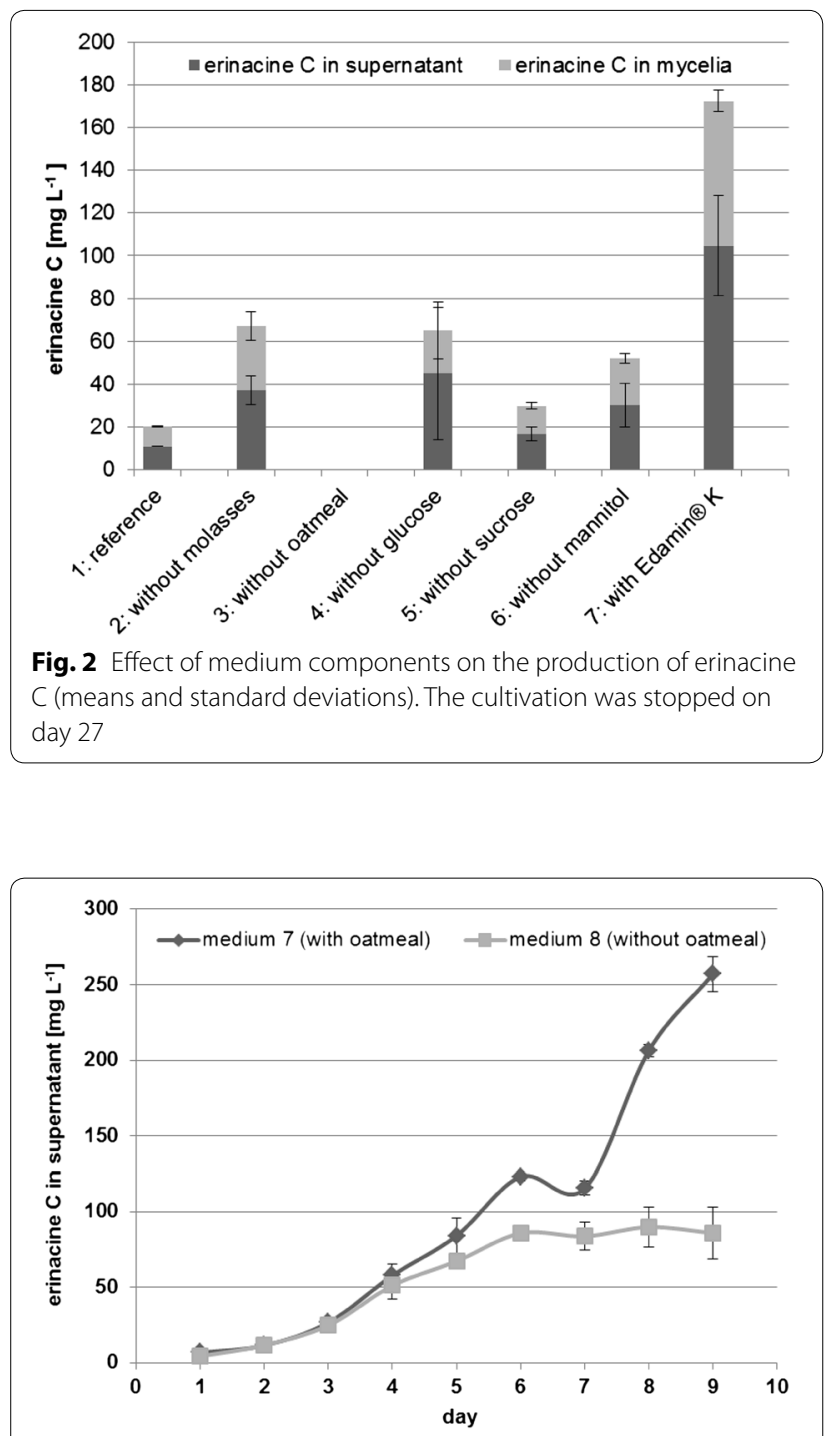

Fig. 3 Production of erinacine $C$ (means and standard deviations) in medium 7 (with oatmeal) versus medium 8 (without oatmeal)

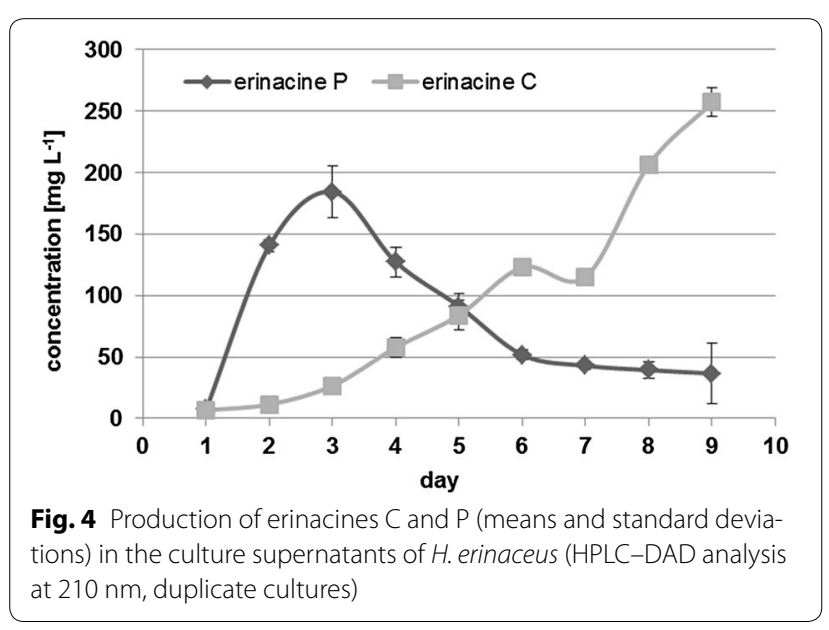

\section{HPTLC-mass spectrometry}

Extracts of mycelia from submerged cultures of $H$. erinaceus on day 4 and the reference compound erinacine $\mathrm{P}$ were both applied on the same HPTLC plate. The MS spectra of the substance zone at $h R_{\mathrm{F}} 34$ showed $m / z$ values of $515.2[\mathrm{M}+\mathrm{Na}]^{+}$and $455.1\left[\mathrm{M}+\mathrm{Na}-\mathrm{CH}_{3} \mathrm{COOH}\right]^{+}$ in the $\mathrm{ESI}^{+}$mode, which were identical with those of the reference compound erinacine P (Fig. 6).

\section{Discussion}

The production of erinacine $\mathrm{C}$ strongly depended on the medium composition. Oatmeal and Edamin ${ }^{\circledR} \mathrm{K}$ supported the formation of erinacine $\mathrm{C}$. Oatmeal as a complex substrate provided protein, fat, carbohydrates, dietary fiber, and mineral nutrients. It might act as a carbon source as well as a nitrogen source. Edamin ${ }^{\circledR} \mathrm{K}$ represents a high quality source of peptides and amino acids produced by enzymatic digestion of lactalbumin, which acted in the medium as a nitrogen source.

The comparison of medium 7 to medium 1 proved the essential role of Edamin ${ }^{\circledR} \mathrm{K}$ for the production of erinacine $C$. The maximum concentration of erinacine $\mathrm{P}$ of $184 \mathrm{mg} \mathrm{L}^{-1}$ was observed after 3 culture days (Fig. 4). Afterwards, the concentrations of erinacine $\mathrm{P}$ decreased while the concentrations of erinacine $C$ steadily increased. These data are in good agreement with the results of Kenmoku et al. [12] who suggested erinacine $\mathrm{P}$ as a precursor of erinacine $\mathrm{C}$. Erinacine $\mathrm{P}$ is supposed to be an early metabolite of the cyathane xylosides family as, e.g., the erinacines $A$ and $B$ were formed from erinacine $\mathrm{P}$. The biosynthetic relationship between erinacine $Q$, erinacine $P$, and erinacine $C$ was elucidated by a $1^{\prime}{ }^{13} \mathrm{C}$-D-glucose labeling experiment. Based on NMR data, $1^{\prime}-{ }^{13} \mathrm{C}$-erinacine $\mathrm{Q}$ was converted into $1^{\prime}-{ }^{13} \mathrm{C}$-erinacine $C$ via $1{ }^{\prime}-{ }^{13} \mathrm{C}$-erinacine $\mathrm{P}[11]$. The erinacines $Q$ and $\mathrm{P}$ may be the common biosynthetic intermediates of cyathane xylosides such as the erinacines $\mathrm{A}, \mathrm{B}, \mathrm{C}$ and $\mathrm{H}$ in $H$. erinaceus, the erinacines $\mathrm{E}, \mathrm{F}$ and $\mathrm{G}$ in $\mathrm{H}$. romosum, and the striatals and striatins in Cyathus spp. However, detailed product formation kinetics of erinacine $\mathrm{P}$ and erinacine $C$ have been reported in the present study for the first time. The product formation kinetics suggested an optimum harvest time of 3 days for erinacine P, and of 9 days for erinacine $C$. The product concentrations of $>250 \mathrm{mg} \mathrm{L}^{-1}$ for erinacine $\mathrm{C}$ could form a basis for a profitable industrial fermentation process.

The production of erinacine $C$ could be further optimized by, e.g., variation of the concentration of Edamin ${ }^{\circledR}$ $\mathrm{K}$, regulation of the culture $\mathrm{pH}$, adjustment of an ideal $\mathrm{C} / \mathrm{N}$ ratio or addition of certain mineral elements. Krzycykowski et al. [18] reported on the effects of the carbon and nitrogen sources, mineral elements, and of the initial $\mathrm{pH}$ of the culture medium on the growth of $H$. erinaceus 
a

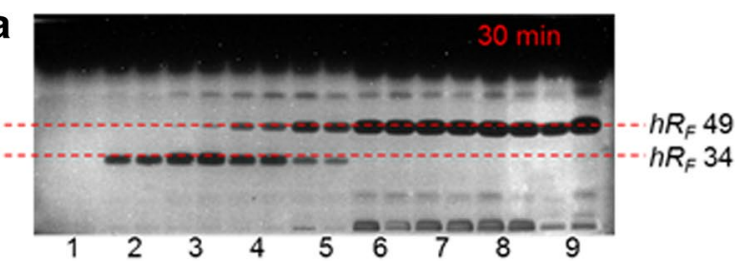

b

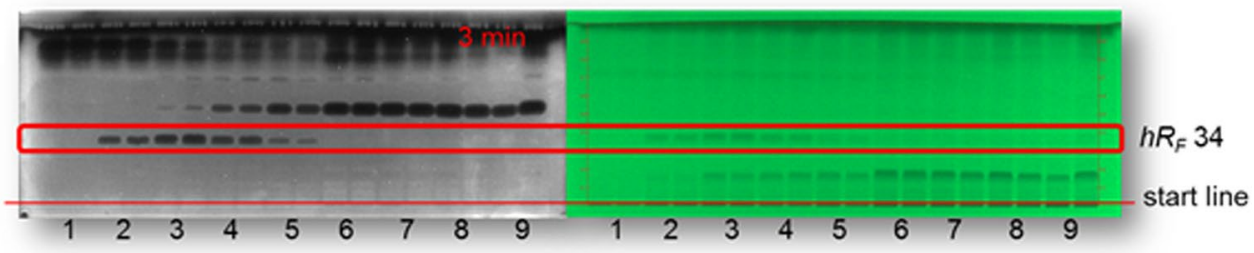

C

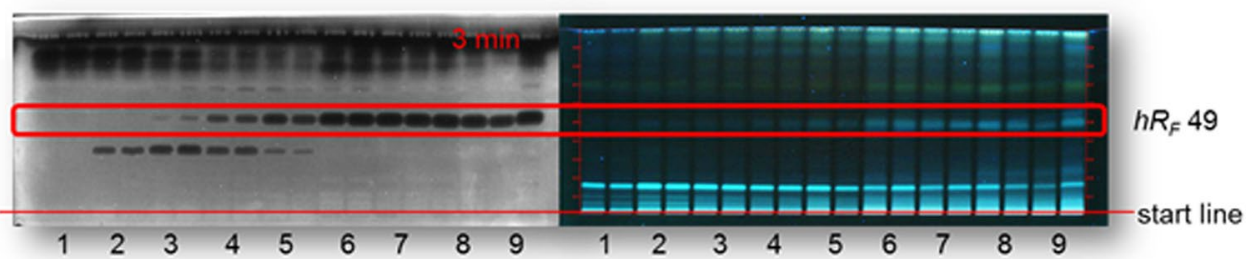

Fig. 5 Direct bioautography (HPTLC-Aliivibrio fischeri) of secondary metabolites from submerged cultures of H. erinaceus from culture days 1-9 (applied in duplicate): a bioluminescent bioautogram documented as grey scale image after $30 \mathrm{~min}$ and after $3 \mathrm{~min}$ compared to detection at $\mathbf{b} \mathrm{UV}$ $254 \mathrm{~nm}$ and $\mathbf{c}$ UV $366 \mathrm{~nm}$

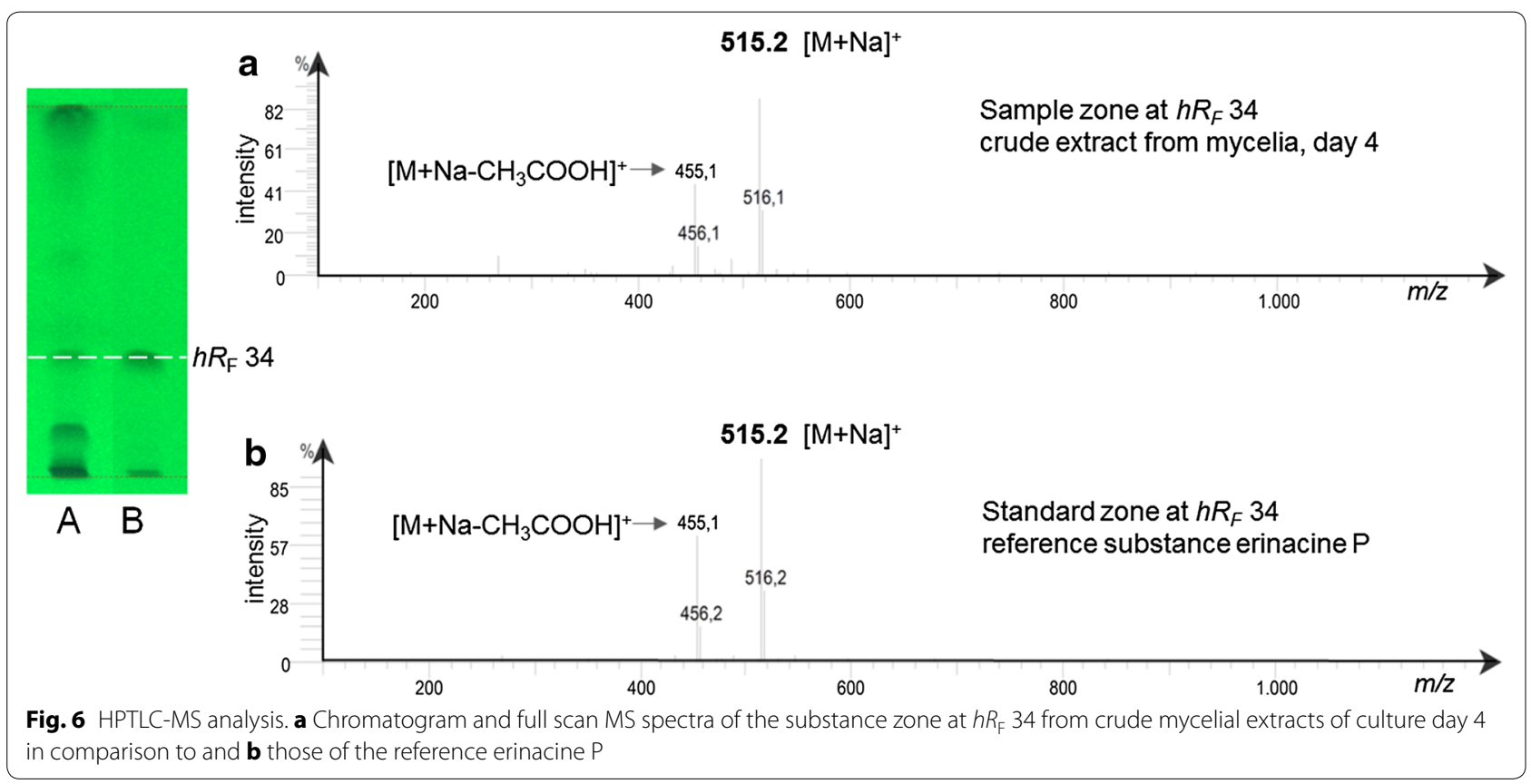

and the production of erinacine $\mathrm{A}$. A more acidic $\mathrm{pH}$ of the culture medium (i.e. $\mathrm{pH} 4.5$ ) positively affected both mycelial growth and erinacine A production. Likewise, the addition of $\mathrm{ZnSO}_{4}$ and $\mathrm{NaCl}$ improved the synthesis of erinacine A. Considering the role of erinacine $\mathrm{P}$ as a precursor of cyathane xylosides, it may be necessary to optimize the yields of erinacine P to achieve higher product concentration of erinacine $\mathrm{C}$. 
HPTLC is commonly used in food analysis to detect, e.g., dyes, sugars, antioxidants, or artificial sweeteners. However, HPTLC is also broadly applied for the analysis of natural products. Recently, isolated sesquiterpenoids from Cyperus rotundus have been screened for their antioxidative potential [19]. The authors described a validated HPTLC method for the identification and quantification of solavetivone, aristolone and nootkatone, which showed no significant variation to the common HPLC method. The combination of HPTLC and bioassays (bioautography) or HPTLC and MS/NMR indicated that HPTLC might be considered as a fast and highly reliable method for compound identification or zone confirmation [20-24].

In the present study, HPTLC-UV/vis/FLD-bioassay was used in addition to HPLC-DAD for the analysis of secondary metabolites from submerged cultures of $H$. erinaceus. While erinacine $\mathrm{C}$ remained at the start line under the chromatographic conditions applied, the substance at $h R_{F} 34$ was confirmed to be erinacine P. This was substantiated by mass spectrometric data, $\mathrm{UV} / \mathrm{vis}$ spectra, and comparison to the authentic reference compound. Comparable product formation kinetics for erinacine P were obtained by HPLC-DAD and HPTLC analyses (Additional file 1: Figures S1, S2). Different from HPLC-DAD, HPTLC allowed for visualization of antibacterial compounds directly on the plate. The antimicrobial activity of erinacine $\mathrm{P}$ has been described previously in [26]. Apart from erinacine $\mathrm{P}$, a second compound with strong activity against Aliivibrio fischeri bacteria $\left(h R_{F} 49\right)$ was detected in the supernatants of $H$. erinaceus. HPTLC-MS analyses did not allow for an unambiguous identification of this compound and of at least two further metabolites with antibacterial activity. Further HPTLC-high-resolution MS studies will be necessary to elucidate the structures of these metabolites.

\section{Conclusions}

Oatmeal and Edamin ${ }^{\circledR} \mathrm{K}$ were found to be essential medium components for the production of erinacine $\mathrm{C}$. The maximum concentration of erinacine $P$ was observed after 3 culture days, while thereafter the concentration of erinacine $C$ steadily increased. The proposed HPTLCUV/vis/FLD-bioassay method allowed for an efficient detection and also quantification of erinacine P. Bioactive secondary metabolites from submerged cultures of $H$. erinaceus were observed instantly via direct bioautography (HPTLC-Aliivibrio fischeri). The direct link to antimicrobial compounds and confirmation by mass spectrometry was considered as streamlined tool for natural product analysis.

\section{Methods}

\section{Fungal strain and reference compounds}

Hericium erinaceus (FU70034, isolated from basidiocarp tissue) was obtained from InterMed Discovery (IMD) Natural Solutions, Dortmund, Germany. The fungus was maintained on a solid medium containing $20 \mathrm{~g} \mathrm{~L}^{-1}$ malt extract (Fluka, Neu-Ulm, Germany) and $15 \mathrm{~g} \mathrm{~L}^{-1}$ agaragar (Roth, Karlsruhe, Germany). The identity of the strain was confirmed by comparison of its ITS nrDNA sequences with reference data in NCBI BLAST ${ }^{\circledR}$ (for sequence data cf. Additional file 1: Figure S3). The strain FU70034 showed $95 \%$ identity to Hericium erinaceus from different geographic origins (Additional file 1: Table S1 with references).

Reference substances of erinacine $\mathrm{C}$ and erinacine $\mathrm{P}$ were isolated from extracts of $H$. erinaceus cultures by preparative HPLC and identified by MS and NMR analyses as described previously [25].

\section{Medium composition and cultivation}

Yeast malt medium consisted of $\mathrm{D}-(+)$-glucose monohydrate $4.0 \mathrm{~g} \mathrm{~L}^{-1}$, malt extract $10.0 \mathrm{~g} \mathrm{~L}^{-1}$, and yeast extract $4.0 \mathrm{~g} \mathrm{~L}^{-1}$ (all Roth). The $\mathrm{pH}$ was adjusted to 6.3 , and the medium was autoclaved at $121{ }^{\circ} \mathrm{C}$ for $20 \mathrm{~min}$ prior to use. Various main culture media were tested (data not shown), and the production of erinacine $\mathrm{C}$ was confirmed only in sugar molasses medium (medium 1). The optimization of erinacine $\mathrm{C}$ production was thus based on medium 1 . One of the carbon sources was omitted in the media $2-7$, respectively, and the nitrogen source Edamin $^{\circledR} \mathrm{S}$ was changed to Edamin ${ }^{\circledR} \mathrm{K}$ in media 7 and 8 (Table 1). Molasses was obtained from Südzucker, Offstein, Germany and oatmeal was from Dr. Oetker, Düsseldorf, Germany. Sucrose and ammonium sulfate were purchased from Roth, and D-Mannitol, Edamin ${ }^{\circledR} \mathrm{S}$, Edamin $^{\circledR} \mathrm{K}$, and calcium carbonate were purchased from Sigma, Taufkirchen, Germany. The media were autoclaved at $121{ }^{\circ} \mathrm{C}$ for $20 \mathrm{~min}$ prior to use.

Pre-cultures of $H$. erinaceus were grown submerged in $250 \mathrm{~mL}$ Erlenmeyer flasks containing $100 \mathrm{~mL}$ yeast malt medium at $24{ }^{\circ} \mathrm{C}$ and $150 \mathrm{rpm}$ for 7 days. Afterwards, $40 \mathrm{~mL}$ homogenized mycelia were inoculated into $400 \mathrm{~mL}$ main culture medium in $1000 \mathrm{~mL}$ Erlenmeyer flasks and incubated at $24^{\circ} \mathrm{C}$ and $150 \mathrm{rpm}$ for further 9 days. All samples for HPTLC analysis were obtained from cultures in medium 7 .

\section{Sample preparation}

Twenty $\mathrm{mL}$ culture supernatant were extracted with $20 \mathrm{~mL}$ ethyl acetate $(\geq 99.5 \%$, Ph. Eur., Roth). The ethyl acetate phase was dried over sodium sulfate (water free, $>99 \%$, Roth) and $15 \mathrm{~mL}$ of the extract was 
Table 1 Main culture media 1-8 for optimization of erinacine $C$ production

\begin{tabular}{lllllllll}
\hline Medium concentration $\left(\mathbf{g ~ L}^{-\mathbf{1}}\right)$ & $\mathbf{1}$ & $\mathbf{2}$ & $\mathbf{3}$ & $\mathbf{4}$ & $\mathbf{5}$ & $\mathbf{6}$ & $\mathbf{7}$ & $\mathbf{8}$ \\
\hline Molasses & 5 & - & 5 & 5 & 5 & 5 & 5 & 5 \\
Oatmeal & 5 & 5 & - & 5 & 5 & 5 & 5 & - \\
D-(+)-glucose monohydrate & 1.5 & 1.5 & 1.5 & - & 1.5 & 1.5 & 1.5 & 1.5 \\
Sucrose & 4 & 4 & 4 & 4 & - & 4 & 4 & 4 \\
D-mannitol & 4 & 4 & 4 & 4 & 4 & - & 4 \\
Edamin ${ }^{\circledR}$ S or K & $0.5 \mathrm{~S}$ & $0.5 \mathrm{~S}$ & $0.5 \mathrm{~S}$ & $0.5 \mathrm{~S}$ & $0.5 \mathrm{~S}$ & $0.5 \mathrm{~S}$ & $0.5 \mathrm{~K}$ & $0.5 \mathrm{~K}$ \\
Ammonium sulfate & 0.5 & 0.5 & 0.5 & 0.5 & 0.5 & 0.5 & 0.5 & 0.5 \\
Calcium carbonate & 1.5 & 1.5 & 1.5 & 1.5 & 1.5 & 1.5 & 1.5 & 1.5 \\
\hline
\end{tabular}

evaporated to dryness. The residue was dissolved in $1 \mathrm{~mL}$ acetonitrile (Chromasolv, gradient grade, Sigma) for HPLC and HPTLC analyses.

Additionally, the concentrations of erinacine $C$ in the mycelia were analyzed by HPLC-DAD. Therefore, the mycelia were separated from the culture supernatants by centrifugation $\left(2880 \times g, 4{ }^{\circ} \mathrm{C}\right.$, and $\left.10 \mathrm{~min}\right)$ and washed 3 times with water. Afterwards, the mycelia were extracted with $20 \mathrm{~mL}$ ethyl acetate twice. The combined ethyl acetate phases were dried over sodium sulfate, and $30 \mathrm{~mL}$ of the extract was evaporated to dryness. The residue was dissolved in $1 \mathrm{~mL}$ acetonitrile for analysis.

Mycelia from culture day 4 were used for HPTLC-MS analyses. The mycelia were prepared as described before, except that $20 \mathrm{~mL}$ of the extract was evaporated to dryness. All experiments were performed in duplicate.

\section{HPLC-DAD analysis}

The HPLC system consisted of pump L-7100, autosampler L-7200, interface D-7000, and diode array detector L-7455 (Merck Hitachi, Darmstadt, Germany). A reversed phase Nucleosil ${ }^{\circledR} 100-5 \mathrm{C}_{18}, \mathrm{CC} 125 / 3 \mathrm{~mm}$ with a respective guard column $(\mathrm{CC} 8 / 3 \mathrm{~mm}$ ) was used (Macherey-Nagel, Düren, Germany). A mixture of acetonitrile (A) (HPLC gradient grade, Sigma) and bidistilled water (B) served as eluent. Gradient: $30 \% \mathrm{~A}(0 \mathrm{~min})-50 \% \mathrm{~A}$ (15 min) $-50 \% \mathrm{~A}(16 \mathrm{~min})-100 \% \mathrm{~A}(23 \mathrm{~min})-100 \% \mathrm{~A}$ (38 $\mathrm{min})-30 \% \mathrm{~A}(43 \mathrm{~min})-30 \% \mathrm{~A}$ (47 $\mathrm{min})$. The flow rate was $0.6 \mathrm{~mL} \mathrm{~min}{ }^{-1}$.

\section{HPTLC-UV/vis/FLD analysis}

HPTLC plates silica gel $60 \mathrm{~F}_{254}, 20 \mathrm{~cm} \times 10 \mathrm{~cm}$, were obtained from Merck, Darmstadt, Germany. Prior to use, the plates were pre-washed with methanol-water $(4: 1, \mathrm{v} / \mathrm{v})$ and dried $\left(110^{\circ} \mathrm{C}, 15 \mathrm{~min}\right)$. The sample extracts were applied as $8 \mathrm{~mm}$ bands onto the HPTLC plate using the Automatic TLC Sampler ATS 4 (CAMAG, Muttenz, Switzerland). The track distance was $10 \mathrm{~mm}$. In the Twin Trough Chamber $20 \times 10 \mathrm{~cm}$ (CAMAG), the plate was developed with $9 \mathrm{~mL} n$-hexane-ethyl acetate-methanol (6:11:1, v/v/v). For an acidic plate conditioning, $1 \mathrm{~mL}$ acetic acid was filled in the opposite chamber trough. After development, the plate was dried at room temperature and documented at UV $254 \mathrm{~nm}$, UV $366 \mathrm{~nm}$, and white light illumination using the TLC Visualizer (CAMAG). The recording of spectra $(200-700 \mathrm{~nm})$ and densitometric absorbance measurement at $210 \mathrm{~nm}$ were performed by TLC Scanner 4 (CAMAG). All data were processed and evaluated by winCATS version 1.4.7.2018 (CAMAG).

\section{Bioautography}

For bioautography, the HPTLC plate was automatically immersed into the bioluminescent Alivibrio fischeri bacteria suspension prepared according to DIN EN ISO 11348-1, Sect. "Methods". The immersion speed was $4 \mathrm{~cm} / \mathrm{s}$ and the immersion time $1 \mathrm{~s}$ using the Chromatogram Immersion Device (CAMAG). The bioautogram was documented as greyscale image with an image accumulation time of $50 \mathrm{~s}$ using the BioLuminizer (CAMAG). Changes were monitored by capturing an image every 3 min over a 30-min period. Alivibrio fischeri bioactive zones were instantly visible as darkened or enlightened zones on the luminescent background.

\section{HPTLC-mass spectrometry}

On the chromatogram, the target zone erinacine $\mathrm{P}\left(h R_{\mathrm{F}}\right.$ 34) was marked at UV $254 \mathrm{~nm}$ with a soft pencil. The TLC-MS Interface equipped with the oval elution head of $4 \mathrm{~mm} \times 2 \mathrm{~mm}$ (CAMAG) was coupled to the electrospray interface of a single quadrupole mass spectrometer (CMS, Advion, Ithaca, NY, USA). The target zone was eluted with methanol at a flow rate of $0.1 \mathrm{~mL} \mathrm{~min}^{-1}$. The total ion current (TIC) full scan mass spectra $(\mathrm{m} / z$ 100$1000)$ were recorded in the positive ionization mode. A plate/system background spectrum was recorded at a comparable migration distance and subtracted from the analyte spectrum. The elution head was flushed with eluent and dried after each measurement. 


\section{Availability of supporting data}

The data sets supporting the results of this article are included within the article and its additional file.

\section{Additional file}

Additional file 1. Comparison of peak areas und UV-VIS spectra obtained for erinacine P by HPLC-DAD and by HPTLC-UV analyses.

\section{Authors' contributions}

TS performed the experiments and drafted the manuscript. GM was responsible for HPTLC analyses and data interpretation and helped to draft the manuscript. $\mathrm{HZ}$ designed the study and helped to draft the manuscript. All authors read and approved the final manuscript.

\section{Author details}

${ }^{1}$ Institute of Food Chemistry and Food Biotechnology, Justus Liebig University Giessen, Heinrich-Buff-Ring 17, 35392 Giessen, Germany. ${ }^{2}$ Institute of Nutritional Science, and Interdisciplinary Research Center for Biosystems, Land Use and Nutrition, Justus Liebig University Giessen, Heinrich-Buff-Ring 26-32, 35392 Giessen, Germany.

\section{Acknowledgements}

Financial support by the excellence initiative of the Hessian Ministry of Science and Art for the LOEWE Center for Insect Biotechnology and Bioresources is gratefully acknowledged.

\section{Competing interests}

The authors declare that they have no competing interests.

Received: 17 November 2015 Accepted: 9 December 2015

Published online: 22 December 2015

\section{References}

1. Kawagishi H, Zhuang C. Compounds for dementia from Hericium erinaceum. Drug Future. 2008;33:149-55.

2. Li G, Yu K, Li F, Xu K, Li J, He S, et al. Anticancer potential of Hericium erinaceus extracts against human gastrointestinal cancers. J Ethnopharmacol. 2014;153:521-30

3. Jiang S, Wang S, Sun Y, Zhang Q. Medicinal properties of Hericium erinaceus and its potential to formulate novel mushroom-based pharmaceuticals. Appl Microbiol Biotechnol. 2014;98:7661-70.

4. Lee KF, Chen JH, Teng CC, Shen CH, Hsieh MC, Lu CC, et al. Protective effects of Hericium erinaceus mycelium and its isolated erinacine A against ischemia-injury-induced neuronal cell death via the inhibition of iNOS/p38 MAPK and nitrotyrosine. Int J Mol Sci. 2014;15:15073-89.

5. Wang M, Gao Y, Xu D, Konishi T, Gao Q. Hericium erinaceus (Yamabushitake): a unique resource for developing functional foods and medicines. Food Funct. 2014:5:3055-64.

6. Ma BJ, Shen JW, Yu HJ, Ruan Y, Wu TT, Zhao X. Hericenones and erinacines: stimulators of nerve growth factor (NGF) biosynthesis in Hericium erinaceus. Mycology. 2010;1:92-8.

7. Kawagishi H, Shimada A, Hosokawa S, Mori H, Sakamoto H, Ishiguro Y, et al. Erinacines $E, F$, and G, stimulators of nerve growth factor (NGF)synthesis, from the mycelia of Hericium erinaceum. Tetrahedron Lett. 1996:37:7399-402.

8. Kawagishi H, Shimada A, Shirai R, Okamoto K, Ojima F, Sakamoto H, et al. Erinacines A, B and C, strong stimulators of nerve growth factor (NGF)synthesis, from the mycelia of Hericium erinaceum. Tetrahedron Lett. 1994;35:1569-72.
9. Kawagishi H, Simada A, Shizuki K, Ojima F, Mori H, Okamoto K, et al. Erinacine D, a stimulator of NGF-synthesis, from the mycelia of Hericium erinaceum. Heterocycl Commun. 1996;2:51-4.

10. Lee EW, Shizuki K, Hosokawa S, Suzuki M, Suganuma H, Inakuma T, et al. Two novel diterpenoids, erinacines $\mathrm{H}$ and I from the mycelia of Hericium erinaceum. Biosci Biotechnol Biochem. 2000;64:2402-5.

11. Kenmoku H, Shimai T, Toyomasu T, Kato N, Sassa T. Erinacine Q, a new erinacine from Hericium erinaceum, and its biosynthetic route to erinacine C in the basidiomycete. Biosci Biotechnol Biochem. 2002;66:571-5.

12. Kenmoku H, Sassa T, Kato N. Isolation of erinacine P, a new parental metabolite of cyathane-xylosides, from Hericium erinaceum and its biomimetic conversion into erinacines $\mathrm{A}$ and $\mathrm{B}$. Tetrahedron Lett. 2000:41:4389-93.

13. Takano M, Umino A, Nakada M. Synthetic studies on cyathins: enantioselective total synthesis of (+)-allocyathin B . Org Lett. 2004;6:4897-900.

14. Trost BM, Dong L, Schroeder GM. Total synthesis of (+)-allocyathin $B_{2}$. J Am Chem Soc. 2005;127:2844-5.

15. Watanabe H, Nakada M. Biomimetic total synthesis of (-)-erinacine E. J Am Chem Soc. 2008;130:1150-1.

16. Wender PA, Bi FC, Brodney MA, Gosselin F. Asymmetric synthesis of the tricyclic core of NGF-inducing cyathane diterpenes via a transition-metalcatalyzed [5+2] cycloaddition. Org Lett. 2001;3:2105-8.

17. Snider BB, Vo NH, O'Neil SV. Synthesis of ( \pm )-allocyathin $B_{2}$ and (+)-erinacine A. J Org Chem. 1998;63:4732-40.

18. Krzyczkowski W, Malinowska E, Herold F. Erinacine A biosynthesis in submerged cultivation of Hericium erinaceum: quantification and improved cultivation. Eng Life Sci. 2010;10:446-57.

19. Priya Rani M, Padmakumari KP. HPTLC and reverse phase HPLC methods for the simultaneous quantification and in vitro screening of antioxidant potential of isolated sesquiterpenoids from the rhizomes of Cyperus rotundus. J Chromatogr B. 2012;904:22-8.

20. Adhami HR, Scherer U, Kaehlig H, Hettich T, Schlotterbeck G, Reich E, et al. Combination of bioautography with HPTLC-MS/NMR: a fast identification of acetylcholinesterase inhibitors from galbanum. Phytochem Anal. 2013;24:395-400

21. Morlock GE, Brett N. Correct assignment of lipophilic dye mixtures? A case study for high-performance thin-layer chromatography-mass spec trometry and performance data for the TLC-MS Interface. J Chromatogr A. 2015;1390:103-11.

22. Meier B, Spriano D. Modern HPTLC - a perfect tool for quality control of herbals and their preparations. J AOAC Int. 2010;93:1399-409.

23. Gössi A, Scherer U, Schlotterbeck G. Thin-layer chromatography-nuclear magnetic resonance spectroscopy - a versatile tool for pharmaceutical and natural products analysis. Chimia. 2012;66:347-9.

24. Naumoska K, Vovk I. Analysis of triterpenoids and phytosterols in vegetables by thin-layer chromatography coupled to tandem mass spectrometry. J Chromatogr A. 2015;1381:229-38.

25. Shen T, Hof LM, Hausmann H, Stadler M, Zorn H. Development of an enzyme linked immunosorbent assay for detection of cyathane diterpenoids. BMC Biotechnol. 2014;14:98.

26. Anke T, Rabe U, Schu P, Eizenhöfer T, Schrage M, Steglich W. Studies on the biosynthesis of striatal-type diterpenoids and the biological activity of herical. Z Naturforsch. 2002:57c:263-71.

\section{Submit your next manuscript to BioMed Central} and we will help you at every step:

- We accept pre-submission inquiries

- Our selector tool helps you to find the most relevant journal

- We provide round the clock customer support

- Convenient online submission

- Thorough peer review

- Inclusion in PubMed and all major indexing services

- Maximum visibility for your research

Submit your manuscript at www.biomedcentral.com/submit
C Biomed Central 